\title{
Analysis of nonlinear parametric vibration of sling considering sling-girder coupling effect
}

\author{
Huihong Shentu ${ }^{\text {a }}$, Zhou $\mathrm{Li}^{\mathrm{b}}$ \\ CHINA CONSTRUCTION STEEL STRUCTURE CORP.LTD, Shenzhen 518048, China \\ a574966949@qq.com, b976112542@qq.com
}

Keywords: Sling, Parametric vibration, Method of multiple scales, Nonlinear, Coupling

\begin{abstract}
Cable has strong nonlinear characteristics under the static or dynamic force, and if the frequency ratio of the boundary excitation meet some conditions, cable under force will perform a vibration amplitude much more than the initial value. The sling of a long-span suspension bridge may perform a parametric vibration with the vibration ratio of 1:1 or 2:1 under the vibrating of bridge deck. This paper, on the problem, considering the first order modal, build a parametric modal that is a sling under bridge deck as the boundary vibration, which is considering the coupling effect between sling and main girder, then deduced the parametric vibration differential equation set which is considering the sling's damping and the coupling effect between the sling and the main girder.
\end{abstract}

\section{Introduction}

The cables of a suspension bridge or a cable-stayed bridge are the major components transferring loads. The bridge deck load of a cable-stayed bridge is transmitted to the main tower by the cables. And on a suspension bridge, it's transmitted to the main tower and the anchorages by slings and the main cable. Cable is a flexible component that is characterized for small bending stiffness, low compressive stiffness, little quality and small damping. So the vibration of cables occurs easily in a variety of excitation which will influence the structural safety and the service life of the bridge. With the increase of span length, the length of the cable is also increasing. Cable has strong nonlinear characteristics under the static or dynamic force. The parametric vibration is one of typical cable nonlinear vibrations which occurs when the frequency ratio of the boundary excitation meet some conditions.

In 1982, Kovacs [1-7] firstly explained the vibration of cables on cable-stayed bridges by the principle of parametric vibration. This text thinks when the bridge deck performs a simple harmonic vibration with a whole-structure bending fundamental frequency $f_{b}$, the cable will perform a transverse vibration with a frequency $f_{c}=f_{b} / 2$. The sling of a long-span suspension bridge is likely to perform a parametric vibration under the vibrating of bridge deck. This paper, on the problem, builds a first order parametric model, deduces the parametric vibration differential equation set which is considering the first two orders, the sling's damping and the coupling effect between the sling and the main girder, and separates variables and reduces the equation set by Galerkin [8] method, then gets suspender's parametric vibration and the response characteristics by using analytical method.

\section{The deduction of the parametric vibration differential equation set which is considering the coupling effect between slings and the main girder}

In a suspension bridge engineering, the main cable, slings and bridge deck are hinged by anchorages. Cable-sling and sling-deck vibration system mainly perform a parametric vibration, which the main cable and the main girder are the boundary excitation origins of, under the load of wind, earth pulsation, vehicle and so on. This paper mainly analyses slings' parametric vibration that is considering the coupling effect between the sling and the main girder. In this paper, the sling is simulated damped uniform massive vertical cable whose upper part is hinged and lower part moves follow the girder. The main girder is simulated a mass block with only vertical displacement. So the 
whole sling-girder system is reduced to the sling-mass block parametric vibration system in Fig1. No consideration of the change of cable force along the length direction, namely ignoring the dead weight of cables;

No consideration of cables' bending stiffness, shear stiffness and torsional stiffness;

Cable is always in linear elastic range in the course of vibration;

Ignoring the spring damping.

Building a parametric vibration that is considering the coupling effect between the sling and the main girder in the coordinate system:

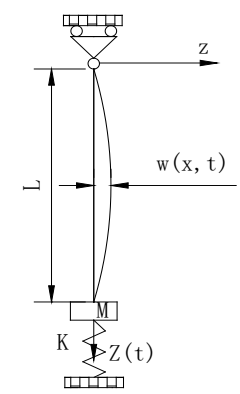

Figure 1.Parametric vibration modal under sling-main girder coupling

This paper builds a parametric vibration model which is considering the damping and the coupling effect between the sling and the main girder as Fig1. In Fig1, $w(x, t)$ is the sling's lateral displacement away from static equilibrium position. $m$ is the quality of per unit length sling. $\rho$ is the damping coefficient of per unit length sling. $L$ is effective length of the sling. $A$ is cross section area of the sling. $E$ is elastic modulus. $T$ is tension. $M$ is mass of the mass block. $Z$ is quality displacement function of time ${ }^{t}$.

Under the coordinate system in Fig1, according Newton's law, it establishes the vibration differential equation of vertical sling as follow:

$$
m \frac{\partial^{2} w(x, t)}{\partial t^{2}}+\rho \frac{\partial w(x, t)}{\partial t}=T \frac{\partial^{2} w(x, t)}{\partial x^{2}}
$$

In Eq.1, $T$ consist of two parts. One is static tension of the sling under the status of static equilibrium, $T_{j}$. As gravity force's distribution along the length direction of the sling is much less than the internal force's at the state of finished bridge, in the basic assumptions, it ignores the influence on sling tension distribution of dead weight and thinks static tension is changeless along the length direction of the sling. The other one is dynamic tension in elastic deformation in the course of vibration, $T_{d}$. And dynamic tension has two parts: elastic dynamic tension caused by boundary axial excitation displacement of the sling and that caused by transverse vibration of the sling.

According to the stress-strain relation of the sling, we know that the dynamic tension of the sling can be expressed as:

$$
T_{d}=E A \frac{Z(t)+\sigma(t)}{L}
$$

In Eq.2, $E$ is elastic modulus of the sling. $A$ is net cross section area of the sling. $Z(t)$ is elastic deformation caused by boundary axial excitation displacement. $\sigma(t)$ is elastic deformation in the course of the sling vibration.

According to transverse displacement of the sling, $\sigma(t)$ is deduced to express as:

$$
\sigma(t)=\int_{0}^{L} \sqrt{1+\left(\frac{\partial w(x, t)}{\partial x}\right)^{2}} d x-L
$$

The transverse deformation of the sling is much less than the axial length of the sling, so $\frac{\partial w(x, t)}{\partial x}$ is much less than 1. In this case, Eq.3 can be reduced by using binomial theorem:

So dynamic tension expression can be expressed as:

$$
\sigma(t)=\frac{1}{2} \int_{0}^{L}\left(\frac{\partial w(x, t)}{\partial x}\right)^{2} d x
$$




$$
T_{d}=\frac{E A}{L}\left(Z(t)+\frac{1}{2} \int_{0}^{L}\left(\frac{\partial w(x, t)}{\partial x}\right)^{2} d x\right)
$$

Supposing a solution of Eq.1 can be written in the modal superposition form[12]:

$$
w(x, t)=\sum_{i=1}^{n} S_{i}(t) \sin (i \pi x / L)
$$

In this equation, $S_{i}(t)$ is the generalized coordinate of amplitude. $\sin (i \pi x / L)$ is mode shape function.

According to the mode shape function in Eq.6, we can see that the odd order modes are symmetric modes and even order modes are dissymmetric modes. In order to accurately understand the response characteristics of the sling, this paper only takes first two order modes to express vibration displacement of the sling:

$$
w(x, t)=S_{1}(t) \sin (\pi x / L)+S_{2}(t) \sin (2 \pi x / L)
$$

Eq.7 is substituted in Eq.4 to get dynamic strain caused by transverse vibration elastic deformation of the sling at the first order mode:

$$
\sigma(t)=\frac{\pi^{2}}{4 L} S_{1}^{2}(t)+\frac{\pi^{2}}{L} S_{2}^{2}(t)
$$

So, the expression of tension $T$ can be written as:

$$
T=T_{j}+T_{d}=T_{j}+E A \frac{Z(t)+\sigma(t)}{L}
$$

$$
=T_{j 0}+\frac{E A}{L}\left(Z(t)+\frac{\pi^{2}}{4 L} S_{1}^{2}(t)+\frac{\pi^{2}}{L} S_{2}^{2}(t)\right)
$$

Then we can get the vibration differential equation of the damped sling:

$$
\begin{aligned}
& m \frac{\partial^{2} w(x, t)}{\partial t^{2}}+\rho \frac{\partial w(x, t)}{\partial t}=\left[T_{j 0}+\frac{E A}{L}(Z(t)\right. \\
& \left.\left.+\frac{\pi^{2}}{4 L} S_{1}^{2}(t)+\frac{\pi^{2}}{L} S_{2}^{2}(t)\right)\right] \frac{\partial^{2} w(x, t)}{\partial x^{2}}
\end{aligned}
$$

And then, Eq.7 is substituted in Eq.10 and dispersed by Galerkin method to get vibration differential equation of no-damped vertical sling:

$$
\begin{aligned}
& m \ddot{S}_{1}(t)+\rho \dot{S}_{1}(t)+\left[T_{j 0}+Z(t)\right. \\
& +\frac{E A \pi^{2}}{4 L^{2}}\left(S_{1}^{2}(t)+4 S_{2}^{2}(t)\right]\left(\frac{\pi}{L}\right)^{2} S_{1}(t)=0 \\
& m \ddot{S}_{2}(t)+\rho \dot{S}_{2}(t)+\left[T_{j 0}+Z(t)\right. \\
+ & \frac{E A \pi^{2}}{4 L^{2}}\left(S_{1}^{2}(t)+4 S_{2}^{2}(t)\right]\left(\frac{2 \pi}{L}\right)^{2} S_{2}(t)=0
\end{aligned}
$$

Eq.10 shows that its form is the same with the vibration equation of linear system's, except that the stiffness term is no longer constant but a function changing with the response. So the whole vibration system is a parametric system and the vibration equation corresponding to it is also parametric equation.

According to Newton's law, the dynamic balance equation of the mass block is established as:

$$
M \ddot{Z}(t)+K Z(t)=-T_{d}
$$

Eq.8 is substituted in Eq.13 to get the vibration differential equation of the mass block:

$$
\begin{aligned}
& M \ddot{Z}(t)+K Z(t)+\frac{E A}{L}\left(Z(t)+\frac{\pi^{2}}{4 L} S_{1}^{2}(t)\right. \\
& \left.+\frac{\pi^{2}}{L} S_{2}^{2}(t)\right)=0
\end{aligned}
$$

Unite Eq.11,Eq.12 and Eq.14, then reduce them to get this equation set:

In the equation:

$$
\left\{\begin{array}{l}
\ddot{S}_{1}(t)+C \dot{S}_{1}(t)+\omega_{1}^{2} S_{1}(t)=-\alpha_{1} S_{1}^{3}(t) \\
-4 \alpha_{1} S_{1} S_{2}^{2}-\alpha_{2} S_{1} Z(t) \\
\ddot{S}_{2}(t)+C \dot{S}_{2}(t)+\omega_{2}^{2} S_{2}(t)=-4 \alpha_{1} S_{1}^{2} S_{2} \\
-16 \alpha_{1} S_{2}^{3}-4 \alpha_{2} S_{2} Z(t) \\
\ddot{Z}(t)+\omega_{2}^{2} Z(t)=-\alpha_{3} S_{1}^{2}-4 \alpha_{3} S_{2}^{2}
\end{array}\right.
$$


$\omega_{1}=\sqrt{\frac{\pi^{2} T_{j}}{L^{2} m}}$, the first order natural frequency without the boundary excitation of the sling; $\omega_{2}$ is the second order natural frequency of the sling, $\omega_{2}=2 \omega_{1}$, the second order natural frequency with the boundary excitation of the sling is twice as the first order frequency.

$\omega_{2}=\sqrt{\frac{K}{M}+\frac{E A}{L M}}$, this expression shows the frequency of mass block includes two parts: the frequency caused by spring-mass system in the case of non-coupling and that caused by sling-mass block system. This verifies the fact that sling-mass block and spring are parallel system.

$\alpha_{1}=\frac{\pi^{4} E A}{4 L^{4} m}$, the coefficient of the cubic nonlinear term, only related to geometry and material property of the sling, known quantity.

$\alpha_{2}=\frac{\pi^{2} E A}{L^{3} m}$, the coefficient of the square nonlinear term, only related to geometry and material property of the sling, known quantity.

$\alpha_{3}=\frac{\pi^{2} E A}{4 L^{2} M}$, the coefficient of the square nonlinear term, related to geometry and material property of the sling, known quantity.

$C=\frac{\rho}{m}$, related to damping ratio and unit mass of the sling, known quantity.

The thought of the method of multiple scales is introducing different time variables which mean different time scales:

$$
T_{n}=\varepsilon^{n} t \quad(\mathrm{n}=0,1,2, \ldots)
$$

So the course of the nonlinear vibration is the function of different scales time variables:

$$
x(t, \varepsilon)=\sum_{n=0}^{m} \varepsilon^{n} x_{n}\left(T_{0}, T_{1}, T_{2} \ldots, T_{m},\right)
$$

In this equation, $m$ is the highest order of small parameter, which depends on computational accuracy. If different scale time variables are considered as independent variables, $x(t, \varepsilon)$ will be the function of $m$ independent time variables. The differential of time can be expanded as $\varepsilon$ power by using composite function differential formula.

$$
\begin{gathered}
\frac{d}{d t}=\frac{\partial}{\partial T_{0}}+\varepsilon \frac{\partial}{\partial T_{1}}+\varepsilon^{2} \frac{\partial}{\partial T_{2}}+\ldots+\varepsilon^{m} \frac{\partial}{\partial T_{m}} \\
=D_{0}+\varepsilon D_{1}+\varepsilon^{2} D_{2}+\ldots+\varepsilon^{m} D_{m} \\
\frac{d^{2}}{d t^{2}}=\frac{d}{d t}\left(\frac{\partial}{\partial T_{0}}+\varepsilon \frac{\partial}{\partial T_{1}}+\varepsilon^{2} \frac{\partial}{\partial T_{2}}+\ldots+\varepsilon^{m} \frac{\partial}{\partial T_{m}}\right)=\left(D_{0}+\varepsilon D_{1}+\varepsilon^{2} D_{2}+\ldots+\varepsilon^{m} D_{m}\right)^{2} \\
=D_{0}^{2}+2 \varepsilon D_{0} D_{1}+\varepsilon^{2}\left(D_{1}^{2}+2 D_{0} D_{2}\right)+\ldots
\end{gathered}
$$

$D_{n}(n=0,1,2, \ldots, m)$ is partial differential operator symbols, defined as:

$$
D_{n} \equiv \frac{\partial}{\partial T_{n}} \quad(n=0,1,2, \ldots, m)
$$

If Eq.18 and Eq.19 are substituted in vibration differential equation; variable ${ }^{X}$ is expanded as Eq.17 and substituted in the differential equation, by comparing the same power coefficients, we can get linear partial differential equations set.

After having eliminated secular terms, these can be obtained:

$$
\left\{\begin{array}{c}
x_{12}=\frac{\alpha_{2}}{\left(\omega_{1}+\omega\right)^{2}-\omega_{1}^{2}} G_{1} G_{3} e^{i\left(\omega_{1}+\omega\right) T_{0}} \\
+\frac{\alpha_{2}}{\left(\omega_{1}-\omega\right)^{2}-\omega_{1}^{2}} G_{1} \bar{G}_{3} e^{i\left(\omega-\omega_{1}\right) T_{0}}+c \\
x_{22}=\frac{4 \alpha_{2}}{\left(2 \omega_{1}+\omega\right)^{2}-4 \omega_{1}^{2}} G_{2} G_{3} e^{i\left(2 \omega_{1}+\omega\right) T_{0}} \\
+\frac{4 \alpha_{2}}{\left(2 \omega_{1}-\omega\right)^{2}-4 \omega_{1}^{2}} G_{2} \bar{G}_{3} e^{i\left(\omega-2 \omega_{1}\right) T_{0}}+c \\
x_{32}=\frac{\alpha_{3}}{4 \omega_{1}^{2}-\omega^{2}} G_{1}^{2} e^{i 2 \omega_{1} T_{0}}+\frac{4 \alpha_{3}}{16 \omega_{1}^{2}-\omega_{2}^{2}} G_{2}^{2} e^{i 4 \omega_{1} T_{0}} \\
-\frac{\alpha_{3}}{\omega^{2}}\left(G_{1} \bar{G}_{1}+4 G_{2} \bar{G}_{2}\right)+c
\end{array}\right.
$$


The subitem of the solution in non-internal resonance condition shows that the sling's response mode shape functions of the first order modal, $e^{i\left(\omega_{1}+\omega\right) T_{0}}, e^{i\left(\omega-\omega_{1}\right) T_{0}}$, are caused by the square nonlinear term which represents the coupling between the first order modal of the sling and the mass block; the response mode shape functions of the second order modal, $e^{i\left(2 \omega_{1}+\omega\right) T_{0}}, e^{i\left(\omega-2 \omega_{1}\right) T_{0}}$, are caused by the square nonlinear term which represents the coupling between the second order modal of the sling and the mass block; the response mode shape functions of mass block, $e^{i 2 \omega_{1} T_{0}}, e^{i 4 \omega_{1} T_{0}}$, are caused by both the first and the second order modal square nonlinear terms of the sling.

Moreover, when $\omega=2 \omega_{1}$, there will be nonsingular solution of the first formula in this equation set. Namely, the excitation frequency of the mass block will lead the first order modal of the sling is internal resonance with the ratio of $2: 1$. When $\omega=4 \omega_{1}$, there will be nonsingular solution of the second formula in this equation set. Namely, the excitation frequency of the mass block will lead the second order modal of the sling is internal resonance with the ratio of 2:1. As the second order frequency is twice as the first order frequency, it can be considered that the excitation frequency of the mass block causes the first order modal of the sling is internal resonance with the ratio of 4:1.

Under these conditions, we can obtained the solution of Eq.22:

$$
\left\{\begin{array}{l}
x_{13}=\frac{1}{8 \omega_{1}^{2}}\left(\alpha_{1}+\frac{\alpha_{2} \alpha_{3}}{4 \omega_{1}^{2}-\omega^{2}}\right) G_{1}^{3} e^{3 i \omega_{1} T_{0}} \\
+\frac{1}{2 \omega_{1}^{2}}\left(\alpha_{1}+\frac{\alpha_{2} \alpha_{3}}{16 \omega_{1}^{2}-\omega^{2}}\right) \bar{G}_{1} G_{2}^{2} e^{3 i \omega_{1} T_{0}} \\
+\frac{1}{6 \omega_{1}^{2}}\left(\alpha_{1}+\frac{\alpha_{2} \alpha_{3}}{16 \omega_{1}^{2}-\omega^{2}}\right) G_{1} G_{2}^{2} e^{5 i \omega_{1} T_{0}} \\
+\frac{\alpha_{2}^{2}}{4\left(\omega^{2}+2 \omega_{1} \omega\right)\left(\omega^{2}+\omega_{1} \omega\right)} G_{1} G_{3}^{2} e^{i\left(\omega_{1}+2 \omega\right) T_{0}} \\
+\frac{\alpha_{2}^{2}}{4\left(\omega^{2}-2 \omega_{1} \omega\right)\left(\omega^{2}-\omega_{1} \omega\right)} \bar{G}_{1} G_{3}^{2} e^{i\left(2 \omega-\omega_{1}\right) T_{0}}+c \\
x_{23}=\frac{1}{2 \omega_{1}^{2}}\left(\alpha_{1}+\frac{\alpha_{2} \alpha_{3}}{16 \omega_{1}^{2}-\omega^{2}}\right) G_{2}^{3} e^{3 i \omega_{1} T_{0}} \\
+\frac{1}{3 \omega_{1}^{2}}\left(\alpha_{1}+\frac{\alpha_{2} \alpha_{3}}{4 \omega_{1}^{2}-\omega^{2}}\right) G_{1}^{2} G_{2} e^{4 i \omega_{1} T_{0}} \\
+\frac{1}{\omega_{1}^{2}}\left(\alpha_{1}+\frac{\alpha_{2} \alpha_{3}}{4 \omega_{1}^{2}-\omega^{2}}\right) G_{2} \bar{G}_{1}^{2} e^{5 i \omega_{1} T_{0}} \\
+\frac{4 \alpha_{2}^{2}}{\left(\omega^{2}+4 \omega_{1} \omega\right)\left(\omega^{2}+2 \omega_{1} \omega\right)} \bar{G}_{2} G_{3}^{2} e^{i 2\left(\omega_{1}+\omega\right) T_{0}} \\
+\frac{4 \alpha_{2}^{2}}{\left(\omega^{2}-4 \omega_{1} \omega\right)\left(\omega^{2}-2 \omega_{1} \omega\right)} G_{2} G_{3}^{2} e^{i 2\left(\omega-\omega_{1}\right) T_{0}}+c \\
x_{33}=\frac{\alpha_{2} \alpha_{3}}{2\left(\omega^{2}+2 \omega_{1} \omega\right)\left(\omega^{2}+\omega_{1} \omega\right)} G_{3} G_{1}^{2} e^{i\left(2 \omega_{1}+\omega\right) T_{0}} \\
+\frac{\alpha_{2} \alpha_{3}}{2\left(\omega^{2}-2 \omega_{1} \omega\right)\left(\omega^{2}-\omega_{1} \omega\right)} \bar{G}_{3} G_{1}^{2} e^{i\left(2 \omega_{1}-\omega\right) T_{0}} \\
+\frac{4 \alpha_{2} \alpha_{3}}{\left(\omega^{2}+4 \omega_{1} \omega\right)\left(2 \omega^{2}+\omega_{1} \omega\right)} G_{2} G_{2}^{2} e^{i\left(4 \omega_{1}+\omega\right) T_{0}} \\
+\frac{4 \alpha_{2} \alpha_{3}}{\left(\omega^{2}-4 \omega_{1} \omega\right)\left(2 \omega^{2}-\omega_{1} \omega\right)} \bar{G}_{3} G_{2}^{2} e^{i\left(4 \omega_{1}-\omega\right) T_{0}}+c
\end{array}\right.
$$

The subitem of mass block's response function shows that in a nonlinear vibration system, the response frequency is much more complex than that in a linear vibration system: besides including each one's own natural frequency, it includes double frequency, sum frequency and difference frequency; the vibration functions of mass block are caused by the sling's first two orders cubic nonlinear terms coupling or the cubic nonlinear terms coupling that represent the sling's first two orders and mass block couple.

Moreover, formula Eq.22 shows when $\omega=\omega_{1}$, there will be nonsingular terms in the first and third formulas in this equation set. Namely, this will lead to the resonance of sling-mass block system. And this resonance is the primary resonance of internal resonances.

For the conditions in the above analysis, that the system performs internal resonances with a ratio of $1: 1,1: 2$ and1:4. 


\section{Conclusions}

By deducing the parametric vibration differential equation set which is considering the sling's damping and the coupling effect between the sling and the main girder, these conclusions can be obtained:

The analytical results of the first two order natural frequency, test results and finite element results verify that the analytical method is correct and suitable for engineering.

When the main girder is the boundary excitation of the sling, if the ratio of excitation frequency and the sling's natural frequency is about 1:1, 1:2 or 1:4, the sling will perform internal resonance with a amplitude that is much larger than its initial excitation value.

Besides 1:1 main resonance, there are 1:2 and 1:4 parametric resonances in the internal resonance of the sling. In these, 1:2 parametric resonance is the sling's first order modal resonance caused by boundary excitations. And the second order modal is influenced very slightly. And 1:4 parametric resonance is the sling's second order modal resonance caused by boundary excitations. But the first order modal is influenced very slightly.

No matter when the main resonance or parametric resonance occurs, there is a strong coupling between the sling and the main girder, and energy transfers between them.

\section{References:}

[1]A.H.Nayfeh,D.T.Mook.Song Jia-su,Luo Wei-de,Chen Shou-ji translate.Nonlinear Oscillations. Higher education press, 1990

[2] Li Fengchen.Parametric Vibration and Cable Force identification of Staying Cable of Large-span Bridges.Harbin Institute of Technology Ph.D. Thesis,2009.12.

[3] 2001.Liu Yan-zhu, Chen Li-qun.Nonlinear Vibrations.Higher education press,2001

[4] 1985.Ji Wen-mei,Fang Tong,Chen Song-qi. Mechanical Vibration.Science Press,1985

[5] Calculation Department of Tongji University .Fundamentals of Numerical Analysis.Tongji University Press, 1998.

[6] I. Kovacs. Zur Frage der Seilschwingungen and der Seildampfung. Die Bautechnik. 1982.

[7] Li Guo-hao.Stability and Vibration of Bridge Structuresl.China Railway Publishing House,1996.

[8] Wang Xu-cheng,Shao Min.The Basic Principle of The Finite Element Method and Numerical Method.Tsinghua Uiversity Press,1997 